\title{
Fundamental Investigation of Si Anode in
}

\section{Li-Ion Cells}

\author{
James J. Wu and William R. Bennett, NASA Glenn Research Center, Electrochemistry Branch
}

\begin{abstract}
Silicon is a promising and attractive anode material to replace graphite for high capacity lithium ion cells since its theoretical capacity is $\sim 10$ times of graphite and it is an abundant element on earth. However, there are challenges associated with using silicon as Li-ion anode due to the significant first cycle irreversible capacity loss and subsequent rapid capacity fade during cycling. In this paper, cyclic voltammetry and electrochemical impedance spectroscopy are used to build a fundamental understanding of silicon anodes. The results show that it is difficult to form the SEI film on the surface of Si anode during the first cycle, the lithium ion insertion and deinsertion kinetics for $\mathrm{Si}$ are sluggish, and the cell internal resistance changes with the state of lithiation after electrochemical cycling. These results are compared with those for extensively studied graphite anodes. The understanding gained from this study will help to design better $\mathrm{Si}$ anodes.
\end{abstract}

Index Terms - Energy Storage - Batteries.

\section{NOMENCLATURE}

Solid electrolyte interphase (SEI)

Lithiation/de-lithiation kinetics

Irreversible capacity

Reversible capacity

Capacity fade

Charge transfer resistance

\section{INTRODUCTION}

NASA is developing ultra-high energy Li-ion cells and batteries for future exploration missions. These cells will utilize advanced anode materials to replace graphite. Silicon is a promising candidate since its theoretical capacity is $\sim 10$ times that of graphite $(1,2)$, and it is an abundant element on earth. However, the use of $\mathrm{Si}$ anodes in Li-ion batteries is challenging due to its significantly lower conductivity and much higher ( 400\%) volume expansion during lithiation and delithiation that occur during the cycling processes (3). Much research has been conducted on $\mathrm{Si}$ anodes aimed at addressing these limitations. Approaches include the addition of carbon fibers or carbon black for improvement of Si conductivity (4, 5), the development of Si/C composite electrodes (6) and the use of nano Si wires for reduction of expansion (7-9). Much progress has been made on these fields.

This study focuses on developing an understanding of the solid electrolyte interphase (SEI) and Li-ion insertion/deinsertion kinetics in cells with silicon anodes. The SEI is a passivating film that is formed on the anode surface by electrolyte decomposition in a lithium ion battery. This film protects the electrolyte solution from further decomposition, and also affects the safety, power capacity, shelf life, cycle life and performance of a lithium-ion battery (10-12). However, the SEI film also limits the capacity and dynamic response of Li-ion batteries by limiting lithium ion transport, and the resistance across the film also restricts the current flow. For optimal performance, the SEI should be highly permeable to lithium ions to minimize the concentration polarization and must be an electronic resistor to prevent SEI thickening that leads to high internal resistance, self-discharge and low faradaic efficiency. The SEI should also be highly ionconductive to reduce overvoltage, and have uniform chemical composition and morphology to ensure homogeneous current distribution. Gaining a fundamental understanding of the phenomena that control the formation of the SEI is essential to the ability to design efficient $\mathrm{Si}$ anodes.

In this article, cyclic voltammetry and electrochemical impedance spectroscopy are used for the investigation of the anode/electrolyte interface, solid electrolyte interphase (SEI) formation and lithium ion transfer kinetics during the electrochemical lithiation and delithiation processes for $\mathrm{Si}$ and graphite anodes. Cyclic voltammetry not only provides the insights into the SEI formation and lithium ion insertion/deinsertion kinetics, but also provides useful information related to the electrochemical stabilities of the electrolyte and electrodes, the presence of any side reactions and the window (range) of potentials used for electrochemical cycling. Cyclic voltammetry is also a useful tool for studying electrode reversibility of charge and discharge and provides a quick prediction of cycling performance. Electrochemical impedance spectroscopy is a non-destructive technique that is useful for investigating the electrode/electrolyte interface and can elucidate SEI formation mechanism and elements such as the resistance and capacitance of the SEI film. The experimental results obtained with $\mathrm{Si}$ anodes are compared with similar assessments of well-established graphite anodes. These studies help develop an understanding of the $\mathrm{Si}$ anode and provide insight into how to address irreversible capacity loss and capacity fade in Si anodes.

\section{EXPERIMENTAL}

\section{A Coin Cell Construction}

Anode Materials, Separator and Electrolyte The $\mathrm{Si}$ anode used in this investigation was developed by NASA industrial partner Lockheed Martin under NASA Research Announcement contract NNC08CB02C that was part of the Enabling Technology Development and Demonstration (ETDD) program, High Efficiency Space Power System (HESPS) battery development effort. The graphite anode for the comparison study was provided by NASA industrial partner Saft America. The graphite electrode was originally coated on both sides, and one side coating was removed for this study. The separator was polyethylene (PE) from Tonen. The electrolyte was $1 \mathrm{M} \mathrm{LiPF}_{6}$ in EC:DEC:DMC $(1: 1: 1 \mathrm{v} / \mathrm{v})$.

Coin Cell Fabrication 2325 type coin cells were fabricated for the electrochemical characterization tests. A thin disk (with 
diameter of 0.5 ") of $\mathrm{Si}$ anode or graphite anode was used as working electrode, and a disk (with a diameter of 0.625 ") of $\mathrm{Li}$ metal was used as the counter electrode. The coin cell was constructed in the following sequence and configuration: positive can, anode disk, separator, Li metal, spacer, spring, and then negative cover. A quantity of $60 \mu \mathrm{l}$ of the electrolyte was added in two steps: the $1^{\text {st }} 30 \mu l$ was added to anode electrode, the separator was stacked on the anode and then the remaining $30 \mu \mathrm{l}$ was added to the separator. Subsequently, the Li metal counter electrode was put on top of the separator, followed by the spacer and spring, and finally the negative cell cover was added and the coin cell was closed. The assembly process for the coin cells was conducted in a glove box filled with Argon. A total of 13 coin cells, with 8 silicon anodes and 5 with graphite anodes, were built for the cyclic voltammetry scan rate tests and impedance measurements.

\section{B. Electrochemical Measurements}

Cyclic Voltammetry $(\mathrm{CV})$ measurements were conducted with an Arbin Instrument BT-2000. For the coin cells with Si anodes, the potential was initially scanned from open circuit voltage $(\mathrm{OCV})$ to $0.05 \mathrm{~V}$ and then back to $1.0 \mathrm{~V}$ for the first cycle, and the scan voltage is set between $1.0 \mathrm{~V}$ and $0.05 \mathrm{~V}$ for the subsequent cycles. Similarly, for the coin cells with graphite anodes, the potential was initially scanned from OCV to $0.01 \mathrm{~V}$, and then back to $1 \mathrm{~V}$ for the first cycle, and for the subsequent cycles, the scan voltage was set between $1 \mathrm{~V}$ and $0.01 \mathrm{~V}$. Scan rates of $1.0,0.5,0.25$, and $0.125 \mathrm{mV} / \mathrm{sec}$ were used in the CV study. A new cell was used for each scan rate test.

Electrochemical Impedance Spectroscopy (EIS) measurements were conducted on a SI 1287 Electrochemical Interface coupling with SI 1260 Impedance/Gain-phase Analyzer. The impedance was measured before and after the cyclic voltammetry tests. The typical frequency range was between $500 \mathrm{kHz}$ to $100 \mathrm{mHz}$ and the applied AC voltage was $10 \mathrm{mV}$. The analysis of the resultant Nyquist plots was conducted with ZPlot/Zview software (Solartron Analytical). The potentials in this paper are referenced to $\mathrm{Li} / \mathrm{Li}^{+}$.

\section{RESULTS AND DISCUSSIONS}

\section{A. SEM Analysis of Anode Electrodes}

The $\mathrm{Si}$ anode and the graphite anode used for these investigations were characterized by SEM. Selected pictures are shown in Figure 1. The $\mathrm{Si}$ anode has a rough and compacted surface with some cracks and agglomerates of $\mathrm{Si}$ and $\mathrm{C}$ particles, as shown in Figure 1A. The sizes of $\mathrm{Si}$ particles are too small to be determined accurately, but most of the particle sizes are $<200 \mathrm{~nm}$, as shown in the high magnification photo in Figure 1B. The graphite anode shown in Figure $1 \mathrm{C}$ has a more even and uniform surface than the Si. The typical graphite particle sizes are in the range of $10 \mu \mathrm{m}$ to $20 \mu \mathrm{m}$, as seen in Figure 1D. The SEM pictures indicate that the graphite anode was probably calendared while the $\mathrm{Si}$ anode was not. The quality and uniformity of the Si anode are not as good as for the graphite anode.
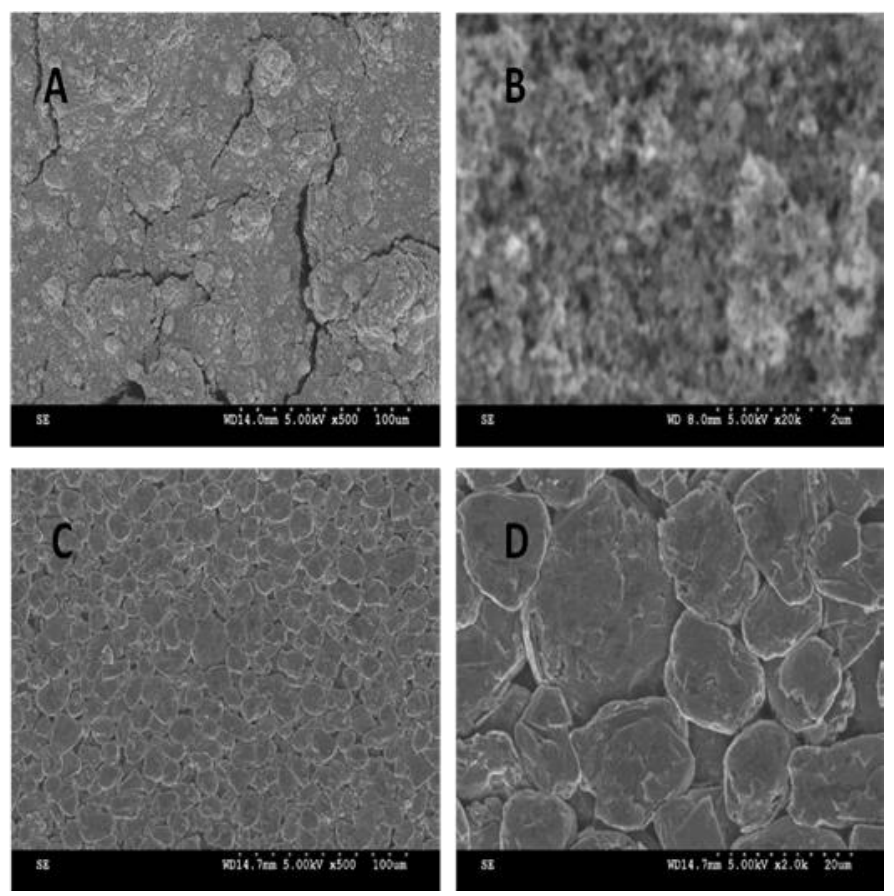

Figure 1 SEM of the $\mathbf{S i}$ anode $(\mathrm{A}, \mathrm{B})$ and the graphite anode $(\mathrm{C}, \mathrm{D})$, Magnification: A 500x; B 20,000x; C 500x; D 2,000x

\section{B. Cyclic Voltammetry Study}

Solid Electrolyte Interphase (SEI) Formation The SEI is a passivating film that is formed on the anode surface by saltmediated electrolyte decomposition. This film protects the electrolyte solution from further decomposition. Cyclic voltammetry was used to study the electrolyte decomposition and SEI formation. As the potential scans cathodically from open circuit voltage (OCV, typical $\sim 3 \mathrm{~V}$ ) to $0.05 \mathrm{~V}$ for the $\mathrm{Si}$ anode at the first cycle, the SEI film starts being formed (at $\sim 0.5 \mathrm{~V}-0.8 \mathrm{~V}$ ) due to the electrolyte decomposition. Ideally, the SEI blocks electrolyte solvents and only the lithium ion can pass through the SEI film and insert into $\mathrm{Si}$ anode (at $\sim 0.2 \mathrm{~V}$ ). This electrochemical lithiation process is reflected by the cathodic current. The potential is cathodically scanned to $0.05 \mathrm{~V}$, and the potential of $0.05 \mathrm{~V}$ is used to avoid the most severe volume expansion region of $\mathrm{Si}$ which occurs at $<0.05 \mathrm{~V}$. As the potential is scanned back to $1.0 \mathrm{~V}$, the inserted lithium ions are de-inserted from $\mathrm{Si}$ anode. This electrochemical deinsertion process is reflected by the anodic current. As shown in Figure 2A, the SEI film for Si anode does not seem to be completely formed in the first cycle since further electrolyte decomposition is observed and the cathodic current continues to increase with cycling. The much lower anodic current vs. the cathodic current in the first cycle indicates less lithium is delithiated, and the charge consumed by electrolyte decomposition in the first cycle for $\mathrm{Si}$ anode results in significant irreversible capacity loss. As the electrochemical cycles increase, the SEI film appears to be slowly formed in the subsequent scans as the cathodic current and anodic current tend to stabilize, as shown in Figure 2A. The result shows that the SEI film is difficult to form for the cells with $\mathrm{Si}$ anode.

The cyclic voltammogram profile for the graphite anode is different from the $\mathrm{Si}$ anode, as shown in Figure $3 \mathrm{~A}$. The 
potential is cathodically scanned to $0.01 \mathrm{~V}$. The potential of $0.01 \mathrm{~V}$ is used to maximize the lithium ion insertion into graphite but to avoid the reduction of the lithium ion to form lithium metal on graphite during the electrochemical lithiation process. The SEI film is formed relatively easily vs. Si anode in the first cycle since the electrolyte decomposition is stopped and the cathodic current does not further increase. The slightly higher cathodic current than the corresponding anodic current at the $1^{\text {st }}$ cycle indicates that there is small irreversible capacity loss at the $1^{\text {st }}$ cycle. Starting from the $3^{\text {rd }}$ cycle, the formed SEI layer is stabilized, as reflected from the stabilized cathodic current from the lithium insertion process and anodic current from de-insertion process.
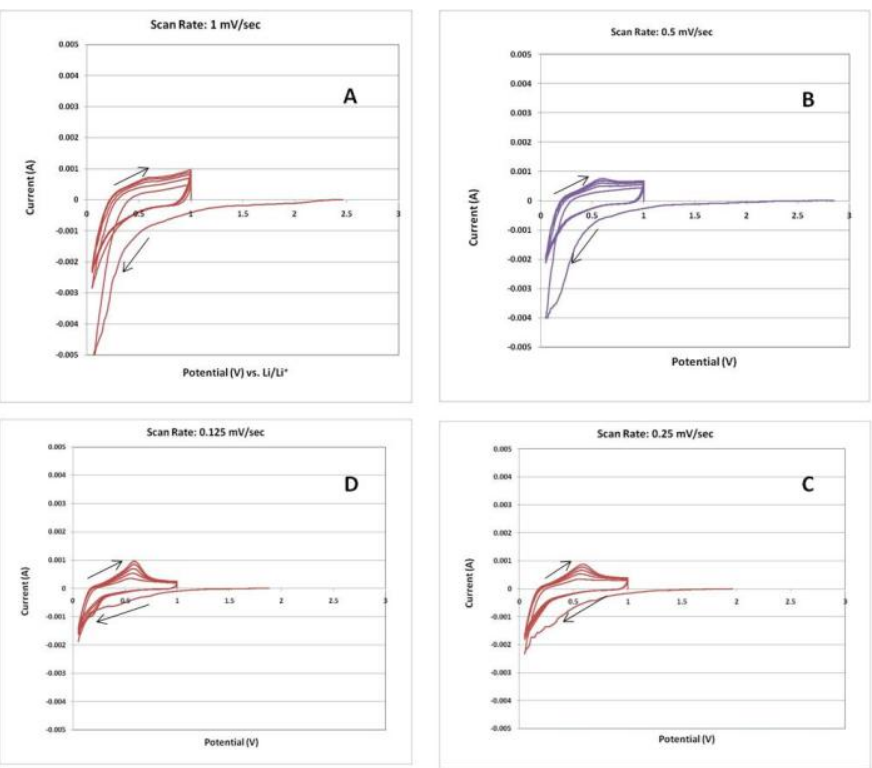

Figure 2 Cyclic voltammograms (the initial 5 cycles) of $\mathbf{S i}$ anode in $\mathrm{Li} / \mathrm{Si}$ coin half-cell with various scan rates: (A) $1 \mathrm{mV} / \mathrm{sec}$; (B) $0.5 \mathrm{mV} / \mathrm{sec}$; (C) 0.25 $\mathrm{mV} / \mathrm{sec}$; (D) $0.125 \mathrm{mV} / \mathrm{sec}$.

The difference between the cyclic voltammetry response for the $\mathrm{Si}$ anodes and graphite anodes is clearly seen. The major difference is in the first cycle. There are several more distinct differences in the cyclic voltammagrams for the $\mathrm{Si}$ and graphite anodes; the $\mathrm{Si}$ anode has poorer reversibility than the graphite anode; the de-lithiation peak $(\sim 0.6 \mathrm{~V})$ for the Si anode is more positive than the de-lithiation peak $(\sim 0.3 \mathrm{~V})$ for the graphite anode at the same scan rate; and the de-lithiation peak for $\mathrm{Si}$ anode is broader than the de-lithiation peak for graphite anode, implying a slow kinetic process of lithium ion insertion/de-insertion in the $\mathrm{Si}$ anode.

Lithium Ion Insertion/De-insertion Kinetics Cyclic voltammetry was also used to study lithium ion insertion/deinsertion kinetics for $\mathrm{Si}$ anode. Figures $2 \mathrm{~A}$ to $2 \mathrm{D}$ are the cyclic voltammograms of $\mathrm{Si}$ anodes at various scan rates of 1 $\mathrm{mV} / \mathrm{sec}, \quad 0.5 \mathrm{mV} / \mathrm{sec}, \quad 0.25 \mathrm{mV} / \mathrm{sec}$ and $0.125 \mathrm{mV} / \mathrm{sec}$, respectively. At the scan rate of $1 \mathrm{mV} / \mathrm{sec}$, not many lithium ions are inserted into the $\mathrm{Si}$ anode, as evidenced by the lack of noticeable anodic current from the delithiation process. As the scan rate decreases, the cathodic current decreases, and the corresponding anodic current increases from the first cycle, which indicates that more lithium ions are inserted into the $\mathrm{Si}$
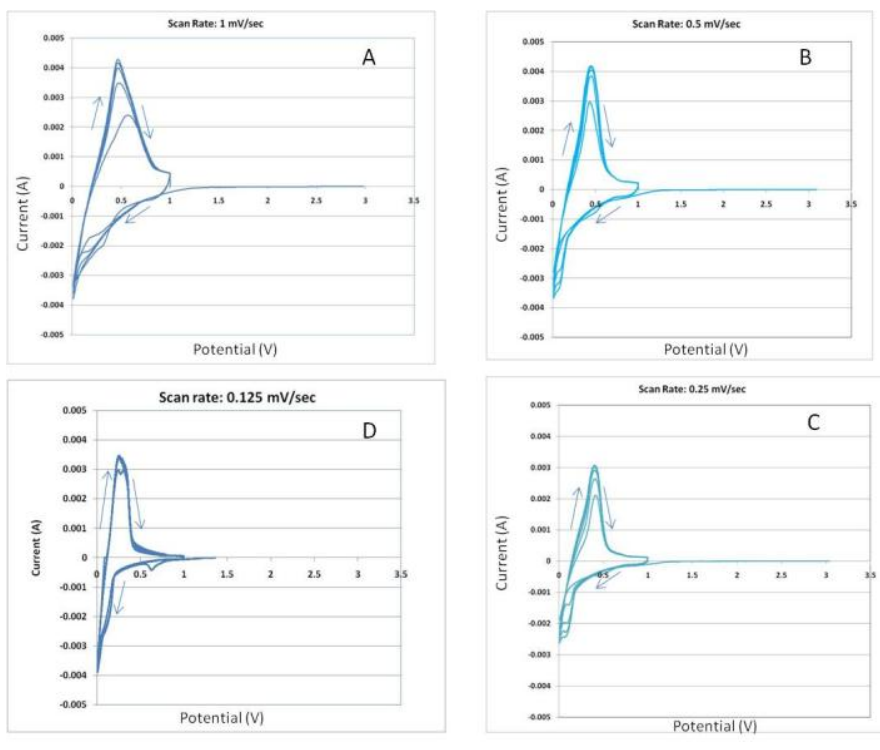

Figure 3 Cyclic voltammograms (the initial 5 cycles) of graphite anode in $\mathrm{Li} / \mathrm{C}$ coin half cell with various scan rates. (A) $1 \mathrm{mV} / \mathrm{sec}$; (B) $0.5 \mathrm{mV} / \mathrm{sec}$; (C) $0.25 \mathrm{mV} / \mathrm{sec}$; (D) $0.125 \mathrm{mV} / \mathrm{sec}$

anode, thus more lithium ions can be de-inserted from the $\mathrm{Si}$ anode. It also shows that the anodic peak (from delithiation process) becomes narrower as the scan rate decreases. All the above observations indicate that the lithium ion insertion and de-insertion to and from $\mathrm{Si}$ anodes is a sluggish process. This agrees with findings from the cyclic voltammetry study of a single Si particle (13). The broader peak width seen for the deinsertion for the $\mathrm{Si}$ anode also implies that the insertion/deinsertion process is slow, which implies that the high rate charge/discharge cycling may be impacted.

The cyclic voltammograms of the graphite anodes at various scan rates, shown in Figures 3A to 3D, are quite different from those for the $\mathrm{Si}$ anode. The de-lithiation peak for graphite anode is much narrower than for the $\mathrm{Si}$ anode. The delithiation peak height for graphite anodes does not change as greatly as seen for the $\mathrm{Si}$ anode. However, the de-lithation peak width for graphite anode decreases as the scan rate decreases. These results indicate that the lithium ion insertion/de-insertion process in graphite anode is faster than the $\mathrm{Si}$ anode.

\section{Electrochemical Impedance Spectroscopy Study}

Electrochemical impedance spectroscopy is a non-destructive and useful tool to study the electrochemical behavior of electrode materials and their interfacial properties, such as resistance and capacitance of SEI film formation. Figure 4A shows the typical impedance spectra for cells with $\mathrm{Si}$ anodes and graphite anodes which were built just before electrochemical cycling. The cells with Si anode and the cells with graphite anode have similar features, i.e. a depressed semi-circle and a tail, implying mixed kinetics and diffusion processes. The equivalent circuit of the cells with $\mathrm{Si}$ anode and the cells with graphite anode are the same, as shown in the top of Figure 4B. The fitted data, which represents the average of at least three cells, shows the corresponding components in the bottom portion of Figure 4B. The results show that the cell internal resistance $R_{i n t}$, which is the sum of electronic 
resistance from the electrode material, separator, contact between active materials with the current collector and the ionic resistance of the electrolyte, is higher for the cell with $\mathrm{Si}$ anode than for the cell with graphite anode. This is reasonable as the $\mathrm{Si}$ itself has lower conductivity than the graphite, and the percentage of active material in the $\mathrm{Si}$ anode is lower than in the graphite anode. Since the Si has a much smaller particle size (mostly <200nm) than the graphite particle (mostly $10 \mu \mathrm{m}$ $-20 \mu \mathrm{m})$, the Si anode has a smaller charge transfer resistance and a higher capacitance than the graphite anode initially.
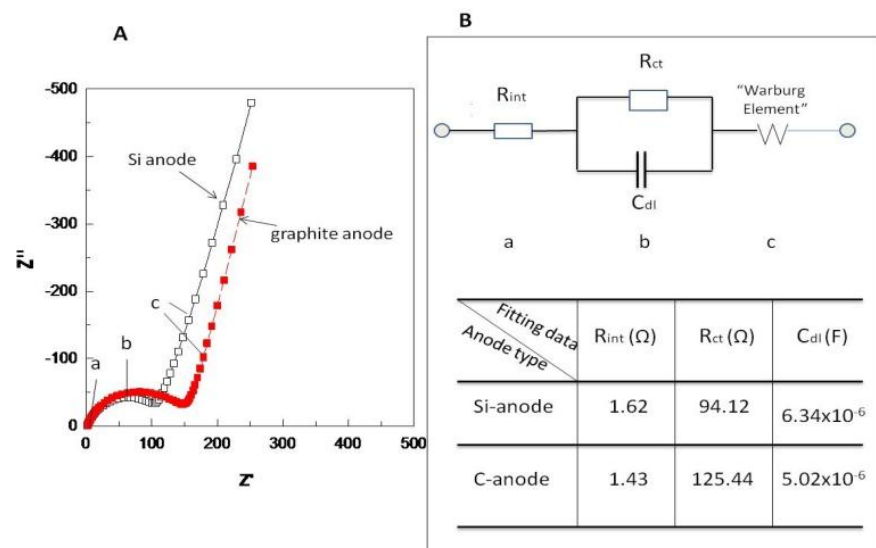

Figure 4 Impedance spectra of comparing cells with $\mathrm{Si}$ anode and graphite anodes before the electrochemical cycling A: typical Nyquist plot; B the equivalent circuit used to fit impedance data, $R_{\text {int }}$ is the cell internal resistance, $\mathrm{R}_{\mathrm{ct}}$ is the charge transfer resistance, $\mathrm{C}_{\mathrm{dl}}$ is the double layer pseudocapacity, and "Warburg Element" is the solid state diffusion element

In combination with the cyclic voltammetry measurement, the impedance was measured after the potential scans to the desired states, such as at $1.0 \mathrm{~V}$ (delithiated state), and at $0.05 \mathrm{~V}$ for Si anode (lithiated state) following the initial $5^{\text {th }}$ cycle for the cells with $\mathrm{Si}$ anode. Figure 5A shows that the impedance spectra before cycling, after scanning to $1.0 \mathrm{~V}$ and after scanning to $0.05 \mathrm{~V}$ for the cells with $\mathrm{Si}$ anodes. The charge transfer resistance of the cell, after electrochemical cycling, is lower than before cycling. This is explained by the fact that the surface of the Si electrode is initially covered with a nonconducting native layer consisting of silicon oxide and silanol (14). After electrochemical cycling, the native layer is broken down, which is possibly caused by the $\mathrm{Li}^{+}$diffusion through the native surface layer, and thus the charge transfer resistance becomes lower. However, the internal resistance, $\mathrm{R}_{\mathrm{int}}$, of the cells after electrochemical cycling is higher than before cycling, as shown in Figure 5B, and this resistance increase is believed to the growth of SEI layer on the $\mathrm{Si}$ electrode. There is a very small, non-obvious depressed semicircle at the high frequency end, which is indicative of the formation of the surface SEI film. In addition, the fitted results

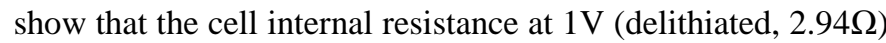
is slightly higher than at $0.05 \mathrm{~V}$ (lithiated, $2.83 \Omega$ ), and both values are much higher than the internal resistance before cycling $(1.74 \Omega)$. This is probably because the volume contraction of the delithiated state (after volume expansion at lithiated state) causes the Si particle shape changes and thus results in loss of $\mathrm{Si}-\mathrm{Si}$ particle contact and causes higher internal resistance.
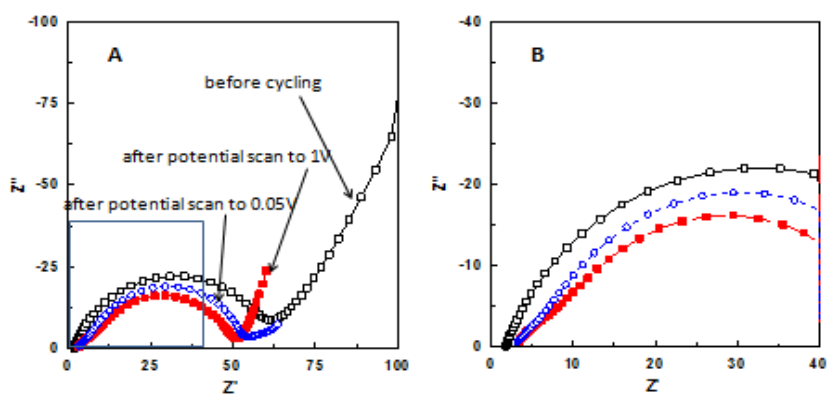

Figure 5 Impedance spectra of the cell with $\mathbf{S i}$ anode $\mathbf{A}$ Nyquist plot; $\mathbf{B}$ the amplification of the square part in $\mathbf{A}$.

Similar procedures were used to analyze the cells with graphite anodes. Figure 6A shows the impedance spectra before cycling, after scanning to $1.0 \mathrm{~V}$ (delithiated state) and after scanning to $0.01 \mathrm{~V}$ (lithiated state) after $5^{\text {th }}$ cycle of cyclic voltammetry measurement. The charge transfer resistance of the cells is significantly reduced after electrochemical cycling. This is due to $\mathrm{Li}^{+}$quick diffusion into the graphite anode. There are two semicircles after scanning to $0.01 \mathrm{~V}$ (lithiated state) as in the cells with the Si anode. The small semicircle at the high frequency end corresponds to SEI film formation. It is interesting to find that there are three semicircles after scanning to $1.0 \mathrm{~V}$ (delithiated state), as shown in Figures 6A and $6 \mathrm{~B}$, indicating multilayer films formed on the graphite anode, which is different from the cells with $\mathrm{Si}$ anodes.

The internal resistance of the cells with graphite anode is higher after cycling than before cycling, which is caused by the growth of the SEI. The fitted results show that the cell internal resistance at $1 \mathrm{~V}$ (delithiated, $1.96 \Omega$ ) is only slightly higher than at $0.01 \mathrm{~V}$ (lithiated, $1.91 \Omega$ ), slightly higher than the internal resistance before cycling $(1.40 \Omega)$. This is because the volume contraction of the delithiated state (after volume expansion at lithiated state) for graphite anode is small, thus the resistance change after lithiation and delithiation process is small.

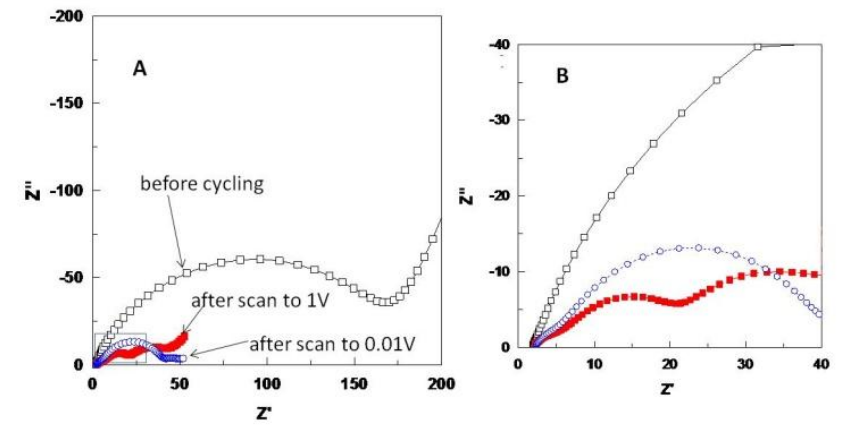

Figure 6 Impedance spectra of the cell with graphite anode A Nyquist plot, $\mathbf{B}$ the amplification of the square part in $\mathbf{A}$. 


\section{CONCLUSION}

Cyclic voltammetry measurements show that the SEI formation on $\mathrm{Si}$ anode is difficult, the $1^{\text {st }}$ cycle irreversible capacity loss is significant and the reversibility is poor for the initial cycles for a $\mathrm{Si}$ anode. However, SEI formation on graphite anodes is easier, the $1^{\text {st }}$ cycle capacity loss is small and the reversibility is good during the initial few cycles. This is probably related to the SEI formation properties. The cyclic voltammetry measurements at various potential scan rates also indicate that the kinetics of the lithium ion insertion and deinsertion processes for the $\mathrm{Si}$ anode are sluggish, while the kinetics for the graphite anode are fast. Impedance spectroscopy measurements further demonstrate that the cell with the $\mathrm{Si}$ anode has a higher initial resistance than the cell with the graphite anode, and the SEI formation results in an increase in the cell internal resistance for both the $\mathrm{Si}$ and graphite anodes. The SEI formed at the $\mathrm{Si}$ anode is more resistive than the SEI formed at the graphite anode. There is also a change in the cell internal resistance between the lithiated state and delithiated state of the $\mathrm{Si}$ anode, but this change is small for the graphite anode. The internal resistance change of the graphite anode is around half of that of Si anode. In addition, the charge transfer resistance after electrochemical cycling for the cell with the $\mathrm{Si}$ anode is approximately twice as high as that for the cell with the graphite anode, implying slow lithium kinetics in the Si anode. The difficult SEI formation, higher initial cell internal resistance, and higher SEI resistance, as well as the greater resistance change during the lithiation and de-lithiation processes may explain the higher irreversible capacity loss and capacity fade for the Si anode.

Future work will evaluate the effects of surface reactive additives to the electrolyte to promote fast SEI formation and to stabilize the SEI on the Si anode. Efforts to optimize the conductive matrix of the $\mathrm{Si}$ anode to lower the volume changes could help to overcome the $1^{\text {st }}$ cycle irreversibility and capacity fade. The combination of cyclic voltammetry with impedance spectroscopy will be useful to evaluate the effectiveness of the suggested modifications on the $\mathrm{Si}$ anode performance.

\section{ACKNOWLEDGMENT}

The authors thank Michelle A. Manzo for her significant effort to input, suggest and revise the manuscript. The authors also thank Eunice Wong for sharing her report for SEM analysis results. The work presented in this paper was performed in support NASA's High Efficiency Space Power Systems (HESPS) Project, which is developing advanced lithium-ion cells for future NASA exploration missions under the Enabling Technology Development and Demonstration (ETDD) Program. This work, which began under the Exploration Technology Development Program (ETDP) Energy Storage Project, combines the efforts of industrial partners and contractors to develop aerospace cell designs with enhanced specific energy and safety characteristics [15].

\section{REFERENCES}

[1] Wen, C. J.; Huggins, R. A. J. Solid State Chem. 1981, 37, 271.

[2] Besenhard, J. O.; Yang, J.; Winter, M. J. Power Sources 1997, 68, 87.
[3] Boukamp, B. A.; Lesh, G. C.; Huggins, R. A. J. Electrochem. Soc. 1981, $128,725-729$.

[4] Xiao, J.; Xu, W.; Wang, D.; Choi, D. W.; Wang, W.; Li, X. L.; Graff, G. L.; Liu, J.; Zhang, J. G. J. Electrochem. Soc. 2010, 157, A1047.

[5] Wolf, H.; Pajkic, Gerdes, T.; Willert-Porada, M. J. Power Sources 2009, 190, 157.

[6] Magasinki, A.; Dixon, P.; Hertzberg, B.; Kvit, A.; Ayala, J.; Yushin, G. Nature Mater. 2010, 9, 353.

[7] Li, H.; Huang, X.; Chen, L.; Wu, Z.; Liang, Y. Electrochem. Solid-State Lett. 1999, 2, 547-549.

[8] Graetz, J.; Ahn, C. C.; Yazami, R.; Fultz, B. Electrochem. Solid StateLett. 2003, 6, A194-A197.

[9] Riccardo R.; Seung, S. H.; Candace, K. C.; Robert, A. H.; Yi, C. J. Phys. Chem. C 2009, 113, 11390.

[10] Peled E., in Lithium Batteries, ed. by Gabano J. P., Academic, 1983, p 43.

[11] Funabiki A.; Inaba M.; Abe T. and Ogumi Z. J. Electrochem. Soc. 1999, 146, 2443-2448.

[12] Peled E.; Golodnitsky D. and Ardel G. J. Electrochem. Soc. 1997, 144, L208.

[13] Chandrasekaran, R.; Magasinski, A.; Yushin, G. and Fuller, T. F. J. Electrochem. Soc. 2010, 157, A1139-A1151.

[14] Yoshio, M.; Wang H.; Fukuda, K.; Umeno, T.; Dimov, N.; and Ogumi, Z. J. Electrochem. Soc. 2002, 149, A1598.

[15] Mercer C. et al., "Energy Storage Technology Development for Space Exploration", NASA/TM-2011-216964

\section{BIOGRAPHIES}

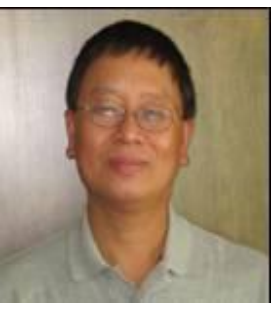

James J. Wu earned his Ph.D. in Chemistry from the University of Illinois at Urbana-Champaign and his Masters degree in Chemistry/Analytical Chemistry from Rutgers University at New Brunswick, NJ. He holds another Masters degree in Electrochemistry/ Electroanalytical Chemistry, and a BS degree in Chemistry/Chemical Engineering. more than 10 years of industrial R\&D experience prior to joining NASA. Dr. $\mathrm{Wu}$ has a varied experience base with the research and development of catalysts, advanced energy storage materials and electrochemical systems, having held positions in industry with various battery development companies that include: Scientist with Wildcat Discovery Technologies, Senior Electrochemical Engineer with PowerGenix, and Staff Technology Engineer with Eveready Battery Company. He possesses a wide range of technical skills that address various aspects of fundamental electrochemical system concepts. Dr. Wu is currently working on Li-ion and Li-metal based battery chemistries, and high energy/high power supercapacitors. His research area of interest includes fundamental understanding electrochemical processes and surface phenomena of electrodes and electrode/electrolyte interface; nanomaterials/nanaocomposites for advanced electrode materials; development of advanced energy storage materials and systems; as well as integration of developmental components for high energy/ultra-high energy battery systems.

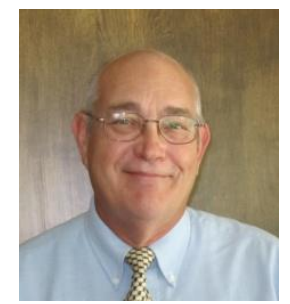

William R. Bennett William Bennett holds a Bachelor's degree in Chemical Engineering. He has worked in the field of electrochemical engineering for over 30 years, building experience in commercial, defense and space applications. Accomplishments have resulted in 7 U. S. Patents and more than 25 technical publications.

Bill has applied the last 11 years of his career supporting a variety of material and battery development projects at the NASA Glenn Research Center (GRC). Bill is currently participating in the development of advanced proton-exchange-membrane (PEM) fuel cell and electrolysis technology for future NASA missions. Current engineering interests include system analysis and water management strategies for PEM fuel cells. 